\title{
Magnetic resonance imaging in the diagnosis of sacral stress fracture
}

\author{
T Featherstone
}

\begin{abstract}
Low back and buttock pain in athletes can be a source of frustration for the athlete and a diagnostic dilemma for the doctor. Sacral stress fractures have been increasingly recognised as a potential cause of these symptoms. As plain radiographs are often normal and the radiation load of an isotope bone scan is substantial, the alternative use of magnetic resonance imaging in the diagnosis of a sacral stress fracture is highlighted in this case report.

(Br F Sports Med 1999;33:276-277)
\end{abstract}

Keywords: stress fracture; back; sacrum; magnetic resonance imaging (MRI)

\section{Case report}

A 25 year old female fitness instructor presented with a 10 day history of unremitting sharp pain in her lower back and right buttock. There was no history of trauma and she was not taking any prescribed or non-prescribed medication. She exercised regularly and ran many miles a week on a treadmill. On examination there was considerable tenderness over the right sacroiliac joint and a small degree of pelvic tilt, with the left side being lower than the right. Movement of the lumbar spine was full and equal in all directions and no abnormality was found in either hip joint. A plain radiograph of the pelvis showed no significant abnormality (fig 1) However, in view of the fact that the severity of her symptoms prevented normal work activity and disturbed her sleep pattern, her general practitioner referred her for magnetic resonance imaging (MRI) of the pelvis. This showed significant bone marrow oedema in the superior aspect of the right sacrum with an associated fracture (fig 2).

The patient subsequently improved with conservative management. A more detailed history from her disclosed several months of amenorrhoea, and her hormonal profile showed low levels of oestradiol, luteinising hormone, and follicle stimulating hormone. She menstruated again following her enforced period of rest.

\section{Discussion}

Stress fractures of the lower extremity and sacrum occur in a variety of people, ranging from

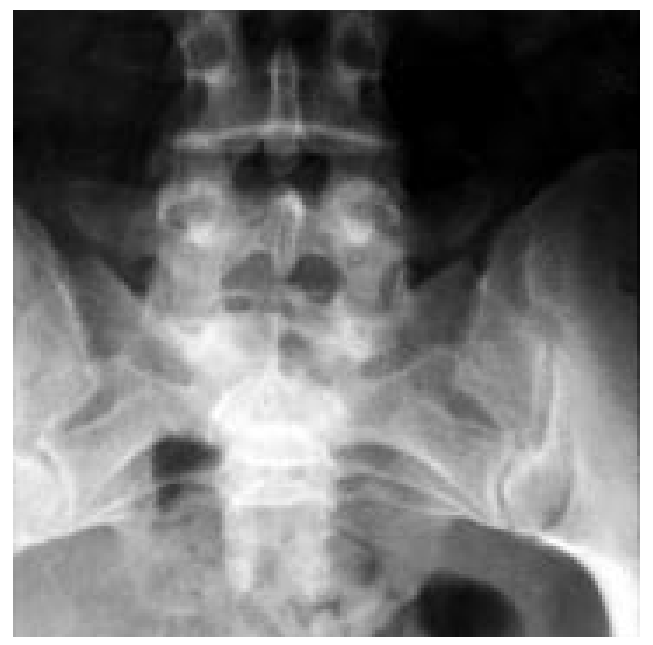

Figure 1 Anteroposterior pelvic radiograph showing no apparent abnormality.

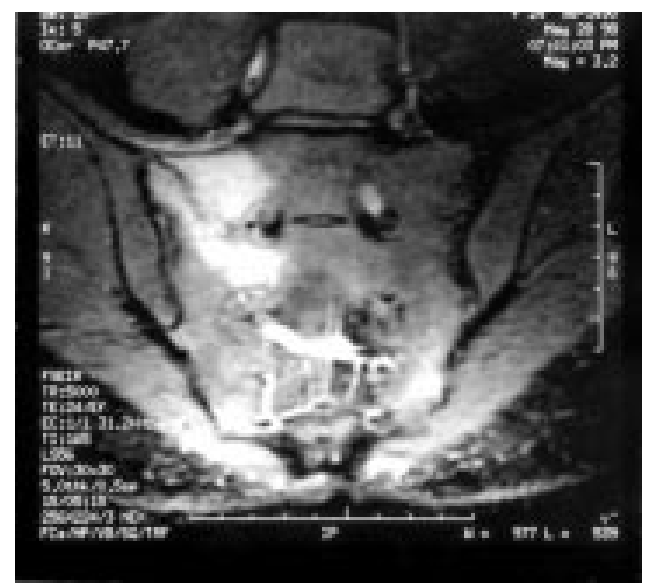

Figure 2 Coronal magnetic resonance fat suppression (STIR) image showing a high signal (white) band of medullary oedema with a fracture line in the right sacral ala.

young healthy athletes to elderly debilitated patients. They can be categorised into two types, fatigue and insufficiency, depending on the state of the bone and nature of the forces applied. Fatigue fractures occur when bones sufficient in mineral content and elasticity are subjected to excessive muscular action or abnormal torque. ${ }^{1}$

Insufficiency fractures may develop when normal physiological stress is applied to bones deficient in mineral content or with abnormal 
elasticity and are common in elderly postmenopausal women with osteoporosis. ${ }^{1}$

Stress fractures are more common in women than men. Women have a lower bone density, lower dietary calcium intake, and a wider pelvis, resulting in different foot plants and running gait which predisposes to an increased risk of fatigue fractures. Menstrual and hormonal irregularities may also be a factor, with a higher incidence of stress fractures in amenorrhoeic athletes as the result of low serum oestrogen and reduced bone density. ${ }^{2}$

The actual incidence of stress fractures in athletes has not been well demonstrated in the past. ${ }^{3}$ Indeed, one of the first case reports of a fatigue stress fracture of the sacrum was only published in $1990 .{ }^{4}$ In 1994 a review of 914 college athletes competing in a variety of sports showed 34 stress fractures of the lower extremities over a two year period. ${ }^{5}$

Plain radiographs are an important first step in the evaluation of suspected fatigue fractures. Unfortunately their yield is limited because patients usually do not have a demonstrable fracture line until about three weeks after symptoms begin. About $67 \%$ of initial radiographs are normal. ${ }^{6}$ Indeed plain radiography of the sacrum may be not only unhelpful but misleading in many cases because of overlying bowel gas and faecal residue.

For many years, three-phase isotope bone scanning has been considered to be the best imaging procedure in the diagnosis of stress fractures. Sensitivity is almost $100 \%$ and scans may be positive as early as 72 hours after the onset of symptoms.

However, MRI is now being used more frequently in the evaluation of suspected stress fractures. The availability of MRI in the United Kingdom has increased significantly in the past few years and accessibility to general practi- tioners has widened its role. In addition, the radiation load to the patient from one bone scan is 4 millisieverts which is equivalent to that from 200 chest $x$ ray examinations! ${ }^{7}$

The bone marrow oedema which is manifested so exquisitely by MRI has the potential to be misinterpreted as neoplasia in certain cases. Fortunately certain characteristic findings on MRI and the appropriate choice of imaging sequence parameters-for instance, fat suppression-does allow fairly reliable differentiation between stress fractures and malignancy even after radiotherapy, although in the presence of multiple stress fractures correct interpretation can become problematic. $^{8}$

In conclusion, this case demonstrates the efficacy of MRI in the diagnosis of a sacral stress fracture. MRI is a safe non-ionising technique, which is an important factor in the investigation of pelvic pathology, particularly in the young patient. Early diagnosis with MRI can lead to quicker management decisions, whereas negative radiographic findings can be falsely reassuring.

1 Pentecost RL, Murray RA, Brindley HH. Fatigue, insufficiency and pathologic fractures. $\mathscr{F} A M A$ 1964;187:111-14. $2 \mathrm{Ha}$ KI, Hahn SJ, Chung M. A clinical study of stress fractures in sports activities. Orthopaedics 1991;14:108995

3 Monteleone GP. Stress fractures in the athlete. Orthop Clin North Am 1995;26:423-32.

4 Bottomley MB. Sacral stress fracture in a runner. Br $\mathcal{F}$ Sports Med 1990:24:243-4.

5 Johnson AW,Weiss CB, Wheeler DL. Stress fractures of the femoral shaft in athletes: more common than expected. Am 7 Sports Med 1994;22:248-56.

6 Mammone JF, Schweitzer MR. MRI of occult sacral insufficiency fractures following radiotherapy. Skeletal Radiol 1995;24:101-4.

7 Royal College of Radiologists London. Making the best use of a department of clinical radiology. Guidelines for Doctors

8 Tyrell PNM, Davies AM. Magnetic resonance imaging appearances of fatigue fractures of the long bones of the lower limb. Br F Radiol 1994;67:332-8.

Take home message

MRI is a safe technique which is now more readily available in the United Kingdom. It has a proven role in the evaluation of suspected stress fractures.

\section{Commentary}

This case study highlights the increasing use of MRI in the diagnosis of sports associated injuries. MRI scanning in patients with unexplained joint pain can lead to an accurate early diagnosis with appropriate management decisions.

With the more widespread use of MRI it is becoming increasingly apparent that occult bone injuries can occur, particularly around weight-bearing joints and that these lesions can mimic soft tissue injuries such as meniscal tears. The recognition of these abnormalities can significantly alter the management of the patient and often avoid invasive procedures as well as unnecessary radiation.

The differential diagnosis has been mentioned to include malignancy, and this is worth remembering as occasionally the reverse scenario can present itself-that is, a patient with known malignancy presenting with bone pain, particularly in the postmenopausal period, can easily have stress fractures and the knowledge of this can avoid erroneous diagnosis of metastatic bone disease. 


\section{Frostbite at the gym: a case report of an ice pack burn}

G O’Toole, S Rayatt

\begin{abstract}
The case is reported of a 59 year old woman who suffered a $1 \%$ total body surface area superficial partial thickness burn to her calf following the application of an ice pack. The cause, resulting injury, and subsequent management are discussed. It is possible that such injuries are common, but no similar reports were found in a literature search. Awareness of the risk of this type of injury is important for all those entrusted with advising patients on the treatment of minor soft tissue injuries.
\end{abstract}

(Br F Sports Med 1999;33:278-279)

Keywords: skin; burns; ice pack

\section{Case report}

A 59 year old woman strained her calf muscles at the gym while running. She was immediately attended to by a member of the gym staff who offered her an ice pack and showed how it should be applied. His erroneous advice was to apply the pack directly to the injured site without any intervening material. For 20 minutes the woman rested the injured limb on the ice pack on a footstool, thus simultaneously compressing and freezing the skin. Over the subsequent 24 hours a large blistered area developed

Department of Burns and Plastic Surgery, Queen Mary's Hospital, Roehampton, London SW15 5PN, United Kingdom G O'Toole

S Rayatt

Correspondence to: Mr G O'Toole, 1 Eastcote View, Pinner, Middlesex HA5 1AT, United Kingdom.

Accepted for publication 5 May 1999

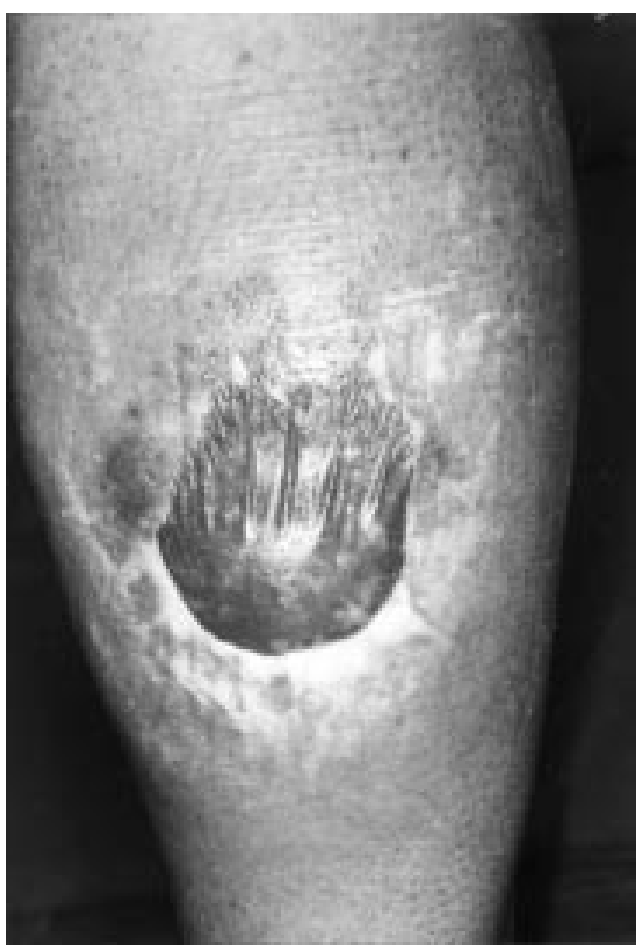

Figure 1 The injury at presentation, four days after the application of the ice pack.

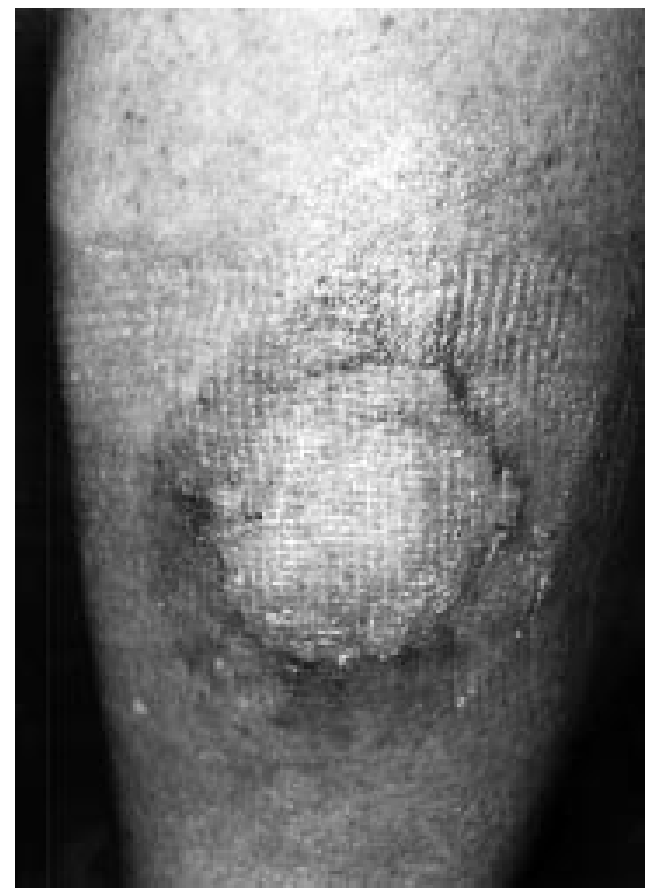

Figure 2 The healed wound after 10 days of treatment.

on the calf, and on the fourth day after the injury the patient presented at casualty (fig 1). A diagnosis of a superficial partial thickness burn covering $1 \%$ total body surface area was made. The initial treatment of this burn was deroofing of its blister. Subsequently the wound was treated conservatively with dressings (vaseline gauze, absorbent gauze, and crêpe dressings), which were changed every second day, until after 10 days the wound had healed (fig 2).

\section{Discussion}

Burns from cold exposure are commonly reported in the literature. However, accidental burns from the application of ice packs to soft tissue injuries are not. Dry ice has been reported to cause such an injury, ${ }^{1}$ but no report of a standard ice pack causing a burn was found in the literature. Cryotherapy-that is, the application of cold in the treatment of injury or disease-is widely used in the treatment of soft tissue injuries. ${ }^{2}$ As no similar reports were found, it was considered of importance to document this case. The risk of litigation is of significance if such an injury were iatrogenic or the result of the advice given by a gym employee. Rest, ice, compression, and elevation (RICE) are standard treatments for musculoskeletal injuries. Direct application of an ice pack to the skin, without intervening 
material, may cause a burn. All those responsible for the treatment of soft tissue injuries must be aware of the consequences of inappropriate application of an ice pack.
1 Gamble WB, Bonnecare ER. Coffee, tea or frostbite? A case report of inflight freezing from dry ice. Aviat Space Environ Med 1996;67:880-1.

2 Swenson C, Sward L, Karlsson J. Cryotherapy in sports medicine. Scand f Med Sci Sports 1996;4:193-200.

\title{
An unusual case of thoracic outlet syndrome associated with long distance running
}

\author{
Y F Leung, O M Chung, P S Ip, A Wong, Y L Wai
}

\begin{abstract}
An amateur marathon runner presented with symptoms of thoracic outlet syndrome after long distance running. He complained of numbness on the $\mathrm{C} 8$ and $\mathrm{T} 1$ dermatome bilaterally. There were also symptoms of heaviness and discomfort of both upper limbs and shoulder girdles. These symptoms could be relieved temporarily by supporting both upper limbs on a rail or shrugging his shoulders. The symptoms and signs would subside spontaneously on resting. An exercise provocative test and instant relief manoeuvre, which are the main diagnostic tests for this unusual case of "dynamic" thoracic outlet syndrome, were introduced. (Br F Sports Med 1999;33:279-281)
\end{abstract}

Keywords: thoracic outlet syndrome; long distance running; marathon running; upper limbs; shoulder girdle

\section{Case report}

A 39 year old male amateur marathon runner presented with sports related numbness and discomfort of both upper limbs and shoulder girdles. The symptoms had been present for three years. He was asymptomatic in normal daily activities and ordinary recreational activities which included badminton and basketball. In 1995 , the patient noticed numbness of the left upper limb on the C8 and T1 dermatome after he had run $10 \mathrm{~km}$. The numbness gradually disappeared over the 10 minutes after he had stopped running. In early 1997, he developed bilateral symptoms after a marathon race. The symptoms persisted for two days. Since that episode, the patient had bilateral symptoms on every occasion after running 10 $\mathrm{km}$. The numbness would vanish within 10 seconds if the upper limbs were supported either on a rail or his own iliac crests. The symptoms would return immediately if the support for the upper limbs was removed.

We initially postulated the cause to be hyperTraumatology Department, Yan Chai Hospital, Tsuen Wan,

NT, Hong Kong, China

Y F Leung

O M Chung

P S Ip

A Wong

Y L Wai

Correspondence to: Dr Y F Leung.

Accepted for publication 11 February 1999 tion in the four limbs, muscle and hand grip power, reflexes, other neurological examination of the upper limbs, Adson's test, neck tilting test,
Halsted (costoclavicular) test, Wright (hyperabduction) test, Roos' test, and provocative tests for other nerve entrapment syndromes.

An on site physical examination was carried out to document the clinical features immediately after $10 \mathrm{~km}$ of running. Two manoeuvres - full extension of both elbows to alleviate entrapment of the ulnar nerve and self controlled ventilation to eliminate factors of hyperventilation-were performed. However, the patient did not show any improvement.

There was diminished touch and pinprick sensation on the C8 and T1 dermatone bilaterally which represented entrapment of the lower brachial plexus. Finally, we discovered that the symptoms and signs would disappear within 10 seconds if both upper limbs were supported on a rail or his iliac crests, or if he shrugged his shoulders.

The $x$ ray picture of the cervical spine was normal. No radiological evidence of cervical rib could be detected. The result of the nerve conduction test was also within the reference range. The $\mathrm{F}$ wave and $\mathrm{H}$ wave were also normal. No other associated peripheral nerve entrapment could be found. Upper limb dexterity functional assessment and hand grip measurement performed by an occupational therapist was also unremarkable at rest.

The patient was diagnosed to be suffering from strenuous exercise induced thoracic outlet syndrome. To our knowledge, it has not previously been reported in the literature. It is an usual case of thoracic outlet syndrome associated with long distance running.

\section{Discussion}

Thoracic outlet syndrome is a well known disease which is due to entrapment of the neurovascular bundle of the upper limbs around the shoulder girdle. It can be classified into arterial, venous, and neurological types of compression. ${ }^{1}$ The first two entities are easily diagnosed and there is objective physical documentation ${ }^{1}$ - for example, an angiogram. ${ }^{2}$ However, the neurological type is vague ${ }^{3}$ and many cases can only be diagnosed by the history and physcial examination. Spiral computed tomography scan of the thoracic outlet is futile for soft tissue compression ${ }^{4}$ because about $70 \%$ of the cases involve soft tissue elements only. Magnetic resonance imaging sometimes offers a better delineation of the compression elements. The sensitivity was $79 \%$, the specificity $87.5 \%$, and the false posi- 
(A)

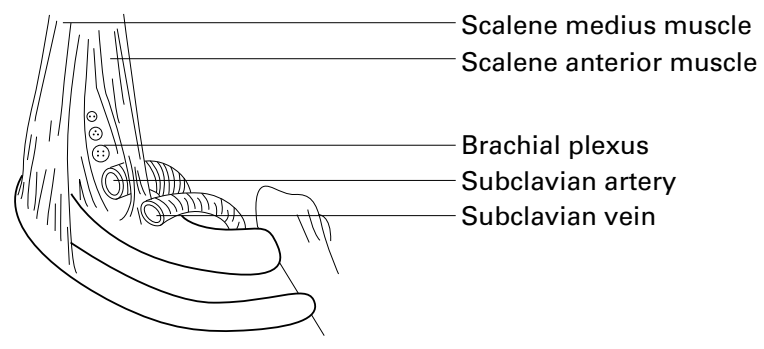

(B)

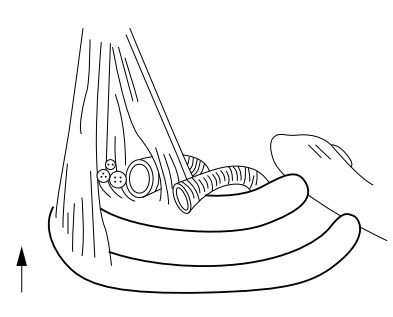

(i)

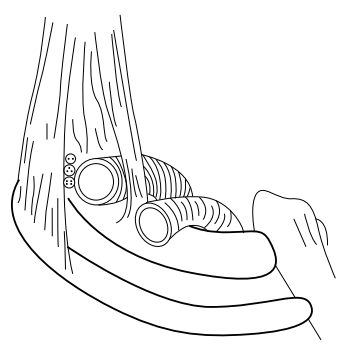

(iii)

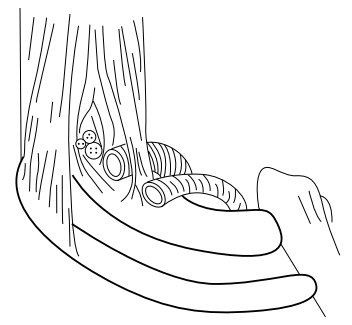

(ii)

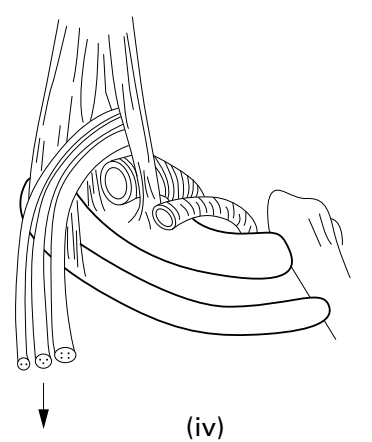

(iv)
Figure 1 Pathoanatomy of dynamic thoracic outlet syndrome. (A) Resting. (B) After exercise: (i) elevation of first rib; (ii) expansion of scalene muscles; (iii) increase in blood flow and the size of the subclavian vessels; (iv) the space for the brachial plexus is narrowed, and the dependent posture of the upper limb exerts traction force on the brachial plexus.

tive rate $9.5 \%$ in one study. ${ }^{5}$ However, the patients were all symptomatic at rest and some of them had neurological deficits including sensation loss and muscle wasting. The role of magnetic resonance imaging is still not well defined. Some authors have suggested that the major use of computed tomography scan and magnetic resonance imaging is to exclude spinal problems or other pathology rather than confirming the diagnosis of thoracic outlet syndrome. ${ }^{6}$ It is also difficult to define the optimal space of the thoracic outlet to accommodate the neurovascular structure.

The compression elements are heterogeneous. They can be fibrous tissue, abnormal cervical rib, callus from fractured clavicle, ${ }^{6}$ abnormal muscle belly and muscle insertion, soft tissue hypertrophy, traumatic fibrosis of soft tissue, ${ }^{7}$ and tumours. However, these patients are all symptomatic, especially when they adopt particular postures with their upper limbs. The symptomatology is considered to be a "static" form: the compression elements are constantly exerting a constrictive effect on the brachial plexus. In contrast, the symptoms of our patient reflect a classical case of thoracic outlet syndrome but they can only be induced by long distance running. The physical findings at rest are all normal.

The mechanism for this unusual "dynamic" thoracic outlet syndrome is still not known. Based on a knowledge of the syndrome, the compression elements are likely to be the dynamic components. The scalene anterior and medius muscles may be responsible. These are also the structures that most commonly produce the neurological type of "static" thoracic outlet syndrome. These muscles are also involved in active and vigorous respiration, being well known accessory respiratory muscles.

The patient may actually have a suboptimal amount of space at the thoracic outlet to accommodate the neurovascular structures. During ordinary activities, the patient may be asymptomatic. However, the venous return and arterial blood flow increase tremendously during strenuous exercise, and the accessory respiratory muscles (scalene muscles) expand because of recruitment of the circulation within them. The other contributing factors may include elevation of the first rib because of hyperventilation and a gravitational effect of the upper limbs on the brachial plexus in the period after exercise. These factors narrow the space significantly at the thoracic outlet and give rise to neurological compression (fig 1). By supporting the upper limbs on a rail or his iliac crests, the patient widens the thoracic outlet space and also eliminates the gravitational effect of the upper limbs on the brachial plexus. $\mathrm{He}$ can then rapidly relieve his symptoms. It explains the pathoanatomy of this unusual case of "dynamic" thoracic outlet syndrome.

Management of the neurological type of thoracic outlet syndrome is mainly conservative, ${ }^{6}$ including shrugging shoulder exercises, posture advice, and training of the shoulder girdle muscles. Operative treatments should be reserved for the resistant cases. Scalenotomy, scalenectomy, excision of the constricting fibrous band, and resection of the first rib are the alternatives. ${ }^{1}$ For a total decompression of thoracic outlet syndrome, a combination of first rib resection and anterior and middle scalenectomy can be considered.

As the symptoms of our patient occurred solely in long distance races, we reassured him and advised an exercise programme, including in particular shrugging shoulder exercises, to strengthen his shoulder girdle muscles. However, there was no significant improvement and he could no longer finish a marathon. The symptoms had neither deteriorated nor improved at a two year follow up. We do not know whether surgical decompression would be beneficial as we have no experience in managing this type of "dynamic" thoracic outlet syndrome. Further follow up should be carried out to monitor progress.

1 Atasoy E. Thoracic outlet compression syndrome. Orthop Clin North Am 1996;27:265-303.

2 Thompson JF, Jannsen F. Thoracic outlet syndrome. Br $\mathcal{F}$ Surg 1996;83:435-6.

3 Novak CB, Mackinnon SE. Thoracic outlet syndrome. Orthop Clin North Am 1996;27:747-62. 
4 Remy-Jardin M, Doyen J, Remy J, et al. Functional anatomy of the thoracic outlet: evaluation with spiral CT. Radiology 1997;205:843-51.

5 Panegyres PK, Moore N, Gibson R, et al. Thoracic outlet syndromes and magnetic resonance imaging. Brain 1993; 116:823-41.
6 Novak CB, Collins ED, Mackinnon SE. Outcome following conservative management of thoracic outlet syndrome. 7 conservative management of tho

7 Casselman F, Vanslenbroek K, Verougstraete L. An unusual cause of thoracic outlet syndrome. F Trauma 1997;43:142-

Take home message

The telltale history, the exercise provocative test, and the instant relief manoeuvre are the diagnostic triad for this syndrome.

\section{Late repair of simultaneous bilateral distal biceps brachii tendon avulsion with fascia lata graft}

Ardi Bayat, Lars Neumann, W Angus Wallace

\begin{abstract}
A 50 year old rock climber sustained a bilateral rupture of the distal biceps brachii tendons. He retained some flexion power in both arms but minimal supination, being weaker on the non-dominant right side. As the patient presented late, with retraction and shortening of the biceps muscle bellies, reconstruction was carried out using fascia lata grafts on both sides. Because of residual weakness onthe left (dominant) side, three further surgical procedures had to be carried out to correct for elongation of the graft.A functionally satisfactory outcome, comparable with that on the right side, was eventually obtained. In summary, bilateral fascia lata grafts to bridge the gap between the retracted biceps bellies and the radial tuberosities were successful in restoring function and flexion power to the elbow. Despite being the stronger side, the dominant arm did not respondas well to the initial surgery. This may be due to overuse of this arm after the operation.

(Br F Sports Med 1999;33:281-283)
\end{abstract}

Keywords: elbow; biceps brachii tendon; avulsion; fascia lata graft; tendon

Avulsion of the distal biceps brachii tendon is an uncommon injury, ${ }^{1}$ and bilateral ruptures are extremely rare. ${ }^{2}$ The incidence varies from 3 to $10 \%$ of all biceps tendon lesions, ${ }^{3}$ and usually occurs at the tendon insertion into the radial bicipital tuberosity. ${ }^{4}$

We present a case of an active male amateur climber with a late presentation of a simultaneous bilateral distal biceps brachii rupture. Several reconstructive procedures were required to repair the defect but a functionally acceptable outcome was finally avhieved.

\section{Case report}

A 50 year old male amateur climber slipped while climbing on a rock face but held on with his fingertips while his elbows were forcefully extended against resistance. He felt a sudden give and extreme pain in both his arms but still held on to the rock. He manoeuvred to a place of safety, and then found he was unable to straighten either arm because of pain and he carried both arms with the elbows flexed.

Rupture of the bicipital tendons was suspected and conservative treatment with analgesia was initiated. He found outdoor activities difficult and even his routine daily tasks were restricted because of weak elbow flexion. He was left hand dominant, but found his right arm weaker than the left, particularly when supinating.

The patient was referred to our clinic two years after the injury. Clinical examination confirmed that he had sustained a detachment of the insertion of both distal bicipital tendons, his right (non-dominant) arm being weaker than the left.

$\mathrm{He}$ underwent a right distal biceps tendon reconstruction, using fascia lata from his right thigh to bridge the defect between the retracted proximal biceps muscle and the distal bicipital tuberosity. After the operation, his right elbow was kept at $90^{\circ}$ of flexion in a collar and cuff for two weeks; this was followed by gentle passive exercises to a maximum of $90^{\circ}$ elbow extension for a further four weeks. He was advised not to do any heavy lifting or pulling with the arms for six months.

Six months later, the right biceps muscle was functioning and the tendon graft was palpable and pulling through with good power. At this stage he was keen to have a similar operation performed on the left side.

Eight months later, he underwent asimilar operation with a fascia lata graftto the left distal biceps tendon. At review five months later, although clinically the distal insertion of the biceps tendon was intact, the result was less satisfactory than that on the right.

He admitted to overuse of the left arm after the operation compared with his right arm despite being given the same advice to avoid overstressing it. He underwent a revision of the reconstructed left biceps tendon and during surgery it was found that his bicipital tendon reconstruction was intact and a thick tendon
Accepted for publication 3 February 1999 


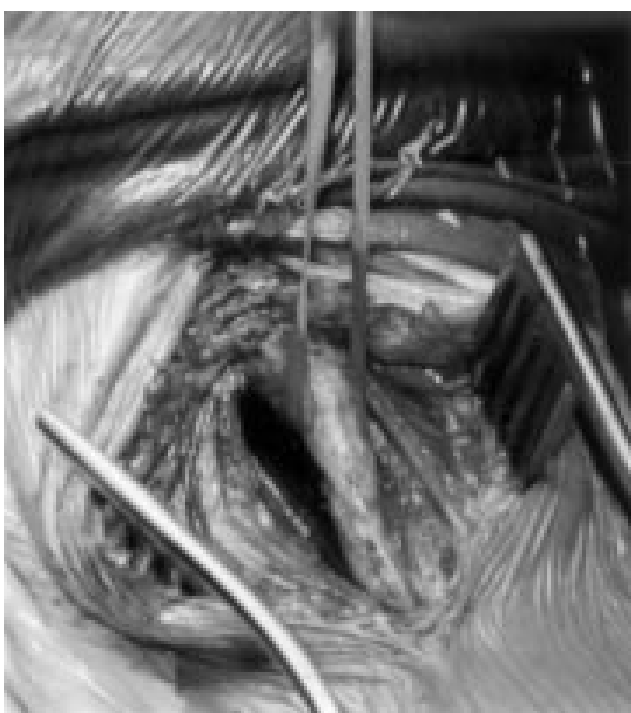

Figure 1 Reconstructed left biceps tendon six months after the operation, at the time of the first plication.

had formed; however, it was elongated and had to be plicated (fig 1).

Despite the revision of the left bicipital tendon, he was still unhappy with the result because of poor flexion and supination power. He continued to have a weak left arm particularly on elbow flexion. In November 1996 he had a further exploration of his left distal biceps, which revealed a thick tendon, which had formed from the graft, but the main (long head) belly of biceps was attached to the anterior elbow capsule and did not take part in elbow flexion. This was reconstructed by weaving the regenerated tendon into the muscle belly.

$\mathrm{He}$ initially had good functional improvement both for elbow flexion and supination, although over the next few months this result deteriorated. On palpation there was a loss of muscle mass at the distal insertion of the bicipital tendon. It was decided to explore for the fourth time in an attempt to improve function. Exploration of the muscle revealed an intact distal tendon with a rupture of the muscle belly at the junction of the middle and distal third of biceps. This defect was repaired with vicryl suture.

At a three month follow up examination, he had a good functional outcome with improved elbow flexion and supination. His subjective functional recovery since this operation has been satisfactory to date.

\section{Discussion}

Bilateral avulsion of the distal biceps brachii tendon is a rare injury. ${ }^{2}$ It has been suggested ${ }^{5}$ that pre-existing degenerative changes in the biceps tendon or its insertion site can cause bicipital tendon rupture. Such ruptures have also been attributed ${ }^{3}$ to degeneration of the tendon secondary to spurs and forced rotation of the radius in relation to the ulna. Patients usually report a single traumatic event such as lifting a heavy load with flexed elbows or a sudden forceful extension of the flexed elbows, as in this case.
Initial management of this injury can be conservative. Non-operative treatment for rupture of the distal biceps tendon has been reported to be associated with no functional deficit, ${ }^{67}$ but others have shown a functional deficit in the biceps if it is left untreated. ${ }^{18}$ In addition, poor cosmesis may result, as the normal contour of the biceps muscle is permanently lost. ${ }^{9}$

Surgical repair is usually carried out for biceps injury in active individuals who require good supination for their sport, hobbies, or vocation and those who do not accept lack of cosmesis. Two main techniques have been proposed, one involving fixation of the tendon to the bicipital tuberosity, ${ }^{10}$ and the other reinsertion into the brachialis muscle. ${ }^{11}$

Several studies ${ }^{12}$ have reported satisfactory results with the Boyd and Anderson ${ }^{10}$ two-incision technique in which the ruptured distal biceps tendon is reattached to the radial bicipital tuberosity using drill holes. ${ }^{12}$ Patients showed a return to normal levels of power in both flexion and supination of the elbow. ${ }^{1}$ Although this technique is currently the trend in managing this disabling condition, it relies upon a tendon of sufficient length to allow insertion into the radial tuberosity. Delayed presentation, proximal retraction, possible degenerative changes, and scarring to neighbouring structures make theoriginal Boyd and Anderson ${ }^{10}$ technique difficult to perform. A new technique using Mitek anchors for reattachment of the biceps tendon to the radial tuberosity has been advocated. ${ }^{13}$

Operative treatment with reattachment of the biceps tendon to the brachialis tendon has produced a variable outcome. ${ }^{9}$ Fascia lata or plantaris longus tendons have been used as grafts for reconstruction of avulsed biceps tendon with good results. ${ }^{28}$

One study using the Boyd and Anderson technique showed that patients with dominant injured extremities had full return of function, whereas in the non-dominant extremity, small deficits in supination and flexion power were noted. ${ }^{14}$ This study found subjective satisfaction with functional outcome in all their patients.

In this case, the non-dominant extremity had full return of function after the repair, whereas the dominant extremity required several attempts to produce a functionally satisfactory outcome.

In conclusion, the use of fascia lata graft to reconstruct the gap between a retracted biceps brachii muscle and the radial tuberosity proved to be successful in restoring function and flexion power to the biceps brachii muscle, particularly on the non-dominant arm. However, in this case aless satisfactory outcome with the dominant arm may have been caused by overuse of that arm in the period after the operation against medical advice, resulting in weakening and failure of the repair. The surgeon may have to warn physically active patients of the possibility of less favourable results if the advice to avoid overstressing the repair is not followed. 
1 Baker BE, Bierwagen D. Rupture of the distal tendon of biceps brachii. I Bone foint Surg [Am] 1985;67: $414-17$

2 Vastamaki M, Brummer H, Solonen KA. Avulsion of the distal biceps brachii tendon. Acta Orthop Scand 1981;52: $45-8$.

3 Gilcrest EL, Albi P. Unusual lesions of muscles and tendons of the shoulder girdle and upper arm. Surgical Gynecology and Obstetrics 1939;68:903-17.

4 Fitzgerald SW, Curry DR, Erickson SJ, et al. Distal biceps tendon injury: MR imagining diagnosis. Radiology 1994; 191:203-6.

5 Davis WM, Yassine D. An etiological factor in tear of the distal tendon of the biceps brachii report of two cases. $\mathcal{F}$ Bone foint Surg [Am] 1956;38:1365-8.

6 Carroll RE, Hamilton LR. Rupture of the biceps brachil: a conservative method of treatment. F Bone foint Surg [Am] 1967;49:10-16.

7 Smith RG, Dooley B, Smith H. Rupture of the distal tendon of the distal biceps brachii tendon. F Bone foint Surg $[\mathrm{Br}]$
8 Morrey BF, Asken L, Kai Nan AN, et al. Rupture of the distal tendon of biceps brachii: a biomechanical study. $\mathcal{F}$ Bone foint Surg [Am] 1985;67:418-20.

9 Potacchini F, Puddu G. Subcutaneous rupture of the distal biceps brachii tendon. $\mathcal{F}$ Sports Med Phys Fitness 1975;15: 81-90.

10 Boyd MM, Anderson LD. A method for reinsertion of the distal biceps brachii tendon. F Bone foint Surg [Am] 1961; 43:1041-3.

11 Dobbie RP. Avulsion of the lower biceps brachii tendon. Analysis of fifty-one previously reported cases. Am f Surg 1941;51:662-83.

12 Agins HJ, Chess JL, Hoekstra DV, et al. Rupture of the distal insertion of the biceps brachii tendon. Clin Orthop $1988 ; 234: 34-8$.

13 Barnes SJ, Coleman SG, Gilpin D. Repair of avulsed insertion of biceps and new technique in four cases. F Bone foint Surg $[B r]$ 1993;75:938-9.

14 Leighton MM, Bush-Joseph CA, Bach Jr BR. Distal biceps brachii repair. Clin Orthop 1995;317:114-21.

\title{
Take home message
}

Bilateral rupture of the biceps brachii tendon is rare. It can be treated conservatively or surgically. In active individuals, operative management can be successful. This may require several reconstructive procedures especially if advice not to overstress the repair is not followed.

\section{A rare fracture-dislocation of the hip in a gymnast and review of the literature}

\author{
J C Mitchell, P V Giannoudis, P A Millner, R M Smith
}

\begin{abstract}
Posterior fracture-dislocation of the hip is an uncommon injury in athletics and leisure activities. It is more commonly seen in high energy motor vehicle accidents and occasionally in high energy sporting activities. A rare case is reported of posterior fracture-dislocation of the hip joint that occurred in a young athlete during gymnastics. This unusual mechanism of injury illustrates the great forces sustained by the hip joint of gymnasts. Early reduction and operative treatment led to a congruent and stable hip joint. After rehabilitation, she returned to light sporting activities after six months.

(Br F Sports Med 1999;33:283-284)
\end{abstract}

Department of

Orthopaedics, St

James's University

Hospital, Leeds,

United Kingdom

J C Mitchell

P V Giannoudis

R M Smith

\section{Department of Trauma and \\ Orthopaedics, University of Leeds P A Millner}

Correspondence to: R M Smith, Department of Trauma and Orthopaedics, St James's University Hospital, Beckett Street, Leeds LS9 7TF, United Kingdom.

Accepted for publication 8 April 1999
Keywords: fracture-dislocation; hip; gymnast; operative treatment

Posterior fracture-dislocation of the hip is usually the result of high energy accidents. Most of the cases are associated with road traffic accidents when the knee strikes the dashboard and the femur is driven posteriorly into the acetabulum. ${ }^{1}$ We present a rare case in which the injury occurred in a 13 year old female gymnast during a practice run up. This type of injury generated by this mechanism has not been reported previously. A review of the literature on fracture-dislocations of the hip joint in sports is also presented.

\section{Case history}

A 13 year old female gymnast presented to our accident and emergency department after an

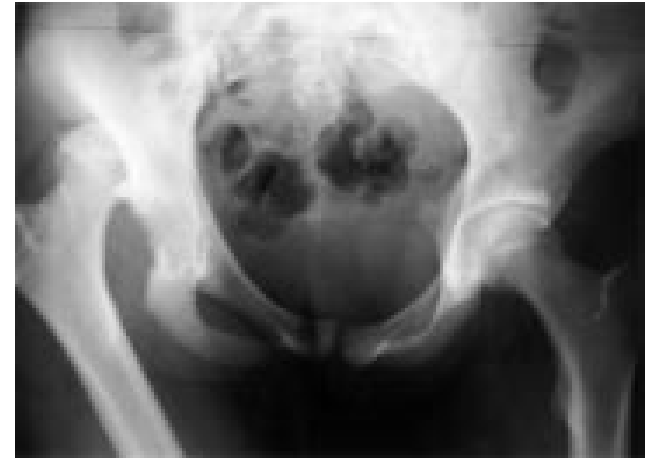

Figure 1 Posterior fracture-dislocation of the hip.

injury to her right hip at school during gymnastics. She had been performing gymnastics for about five years. She was 5 feet 6 inches tall and weighed 110 pounds. She reported that she was practising her run up before attempting a vault and landed one footed on a springboard left at an incline of $45^{\circ}$ against the far side wall of the gymnasium. At the time of impact, the hip was flexed with a one foot impact. The hip was then adducted and internally rotated around the planted foot, resulting in sudden pain in the hip and an inability to bear any weight.

On examination, the right leg was found to be clinically dislocated posteriorly, the sciatic nerve was intact, and there was no evidence of vascular injury. Plain radiographs disclosed a posterior fracture-dislocation of the right hip (fig 1). There was no history in the family of any connective tissue disease or joint hypermobility. An urgent closed reduction under general anaesthesia was performed. Computed tomography scan illustrated a congruent re- 


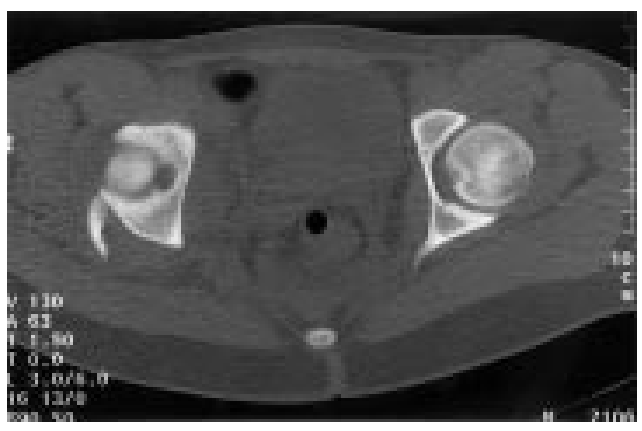

Figure 2 Computed tomography scan of the hip joint showing a congruent reduction of the joint with an associated posterior wall fracture.

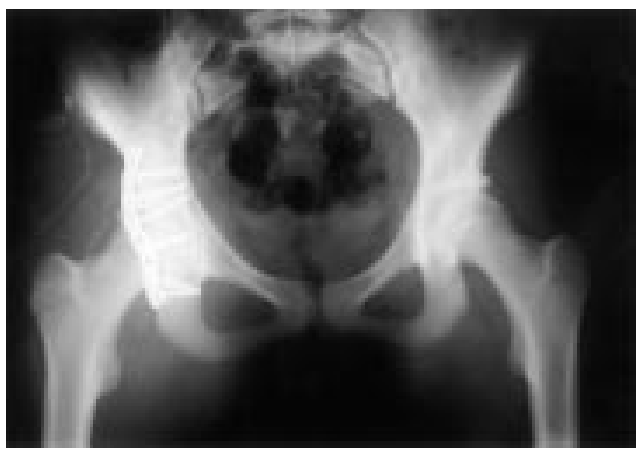

Figure 3 Radiograph of hip after stabilisation of the fracture with lag screws and a buttress plate.

duction of the principal part of the joint with an associated posterior wall fracture (fig 2 ). There was no significant marginal impaction.

A standard posterior wall acetabular reconstruction was performed using a KocherLangenbeck (posterior) approach. The fracture was stabilised by lag screws and a buttress plate (fig 3). The patient was mobilised, touch weight bearing on the third day after the operation, and discharged home uneventfully on the sixth day after the operation with the aid of crutches. Follow up at 12 months was satisfactory, with the patient able to move without pain and the aid of crutches; she had already returned to light sporting activities six months after the injury. Follow up radiographs of the joint showed maintenance of joint congruity and no evidence of avascular necrosis of the femoral head or heterotopic ossification.

\section{Discussion}

Posterior wall fractures of the acetabulum account for about a third of the cases in most series. ${ }^{2}$ More than $70 \%$ of these injuries are sustained in high energy road traffic accidents; the incidence of such injuries is said to parallel the degree of energy transfer in these accidents. $^{3}$ The incidence of such injuries in sport is much lower; ${ }^{4}$ although they have been well documented in American football, rugby, skiing, horse riding, cycling, and even jogging, ${ }^{5-8}$ we cannot identify a previous report of this injury occurring during gymnastics.

The normal mechanism is an axial force along the femur with the hip flexed. This is usually applied by impact on a dashboard or the like during a rapid deceleration road traffic accident. In our case the gymnast had the hip flexed, adducted, and internally rotated at the time of injury. The inclination of the springboard was greater than normal for a vault and we believe that this contributed to the injury pattern. Letournel and Judet ${ }^{9}$ showed that the posterior acetabular rim bears the impact from the femoral head in this position. The mechanism will be the same in this case; laboratory models have shown that the joint reaction force during the stance phase of a running gait can reach up to five times the body weight ${ }^{10}$ and is likely to be even greater at the impact of a gymnastic vault.

Traumatic hip dislocations in children and adolescents have also been described, ${ }^{11}$ but these are rarely associated with fracture.

Indications for operative management of this type of injury include significant articular displacement (more than $2 \mathrm{~mm}$ ), instability of the joint after closed reduction, irreducibility of the hip, neurovascular injury, and ipsilateral femoral fracture. ${ }^{12}$

In our case operative intervention produced a stable congruent joint; after 12 weeks of restricted weight bearing, the gymnast increased her activity. After rehabilitation, she was able to return to light sporting activities six months later.

Contributors: P G initiated and coordinated the collection of information. J M interviewed the patient and wrote the paper with P G. P M participated in the design and editing of the paper. M S performed the operative procedure and participated in the design and editing of the paper.

1 Jacob JR, Rao JP, Ciccarelli C. Traumatic dislocations and fracture dislocation of the hip: a long-term follow-up study. Clin Orthop 1987;214:249-63.

2 Letournel E. Acetabulum fractures: classification and management. Clin Orthop 1980;151:81-106.

3 DeLee JC. Fractures and dislocations of the hip. In: Rockwood CA Jr, Green DP, eds. Fractures in adults. 3rd ed. Philadelphia: JB Lipincott Co, 1991;2:1481-652.

4 O'Donoghue DH. Treatment of injuries to athletes, 4 th ed. Philadelphia: WB Saunders Co, 1984:427-8.

5 O'Leary C, Doyle J, Fenelon G, et al. Traumatic dislocation of the hip in rugby union football. Ir Med f 1987;80:291-2.

6 Stanisavljevic S, Irwin RB, Brown LR. Orthopaedic injuries in water skiing: etiology and prevention. Orthopaedics 1978; 1:125-9.

7 Bass A, Lovell ME. Two cases of acetabular fractures sustained during competitive cycling. $\mathrm{Br}$ f Sports Med sustained during

8 Wolfe MW, Brinker MR, Cary GR, et al. Posterior fracturedislocation of the hip in a jogger. $\mathcal{F}$ South Orthop Assoc 1995;4:91-5.

9 Letournel E, Judet R. Fractures of the acetabulum. Berlin, Springer-Verlag, 1981:7-12.

10 Rydell N. Biomechanics of the hip joint. Clin Orthop 1973;92:6-15.

11 Pearson DE, Mann RJ. Traumatic hip dislocation in children. Clin Orthop 1973;92:189-94.

2 Kuner EH. Indication, technique and complications in the surgical treatment of acetabular fractures. Orthopade 1997; 26:327-35.

\section{Take home message}

Posterior wall fractures of the acetabulum are not common in sports. In gymnastics care should be taken to avoid performing a vault or practising a run up when the inclination of the springboard is greater than anticipated. Operative intervention is required to produce a congruent joint. 